\title{
Concepciones modernas sobre la naturaleza y la sociedad: el legado del pensamiento de Marx
}

\author{
Salvador Orlando Alfaro
}

Universidad de Regina, Canadá

RESUMEN: El presente trabajo se centra en discutir algunas ideas fundamentales en el pensamiento de Marx para entender las condiciones actuales de la relación entre la sociedad y la naturaleza. Al mismo tiempo, presenta su teoría como una consecución y refinamiento de las ideas del iluminismo y muestra cómo, a partir del uso de categoría del trabajo humano, Marx es capaz de presentar una interpretación materialista de las relaciones dinámicas entre la sociedad y el medio ambiente.

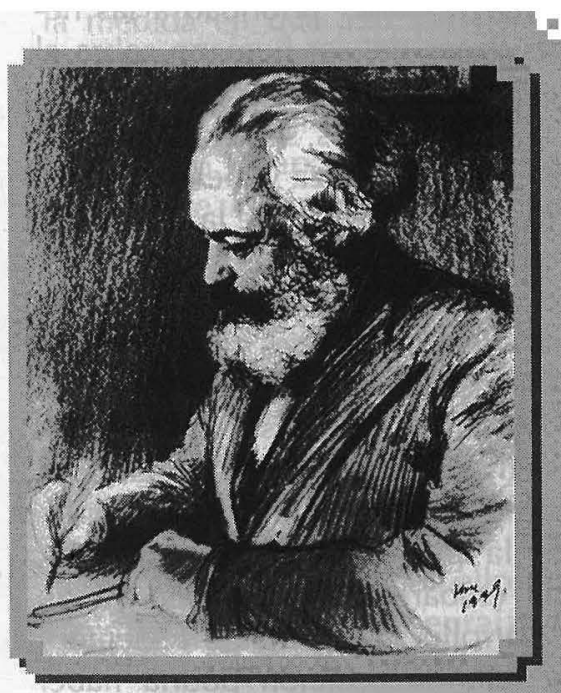

ABSTRACT: The following essay is focused on some fundamental ideas on Marxist thought in order to understand the current conditions of the relation between society and nature. On the same time, it shows Marxist theory as a consecution and refinement of the Enlightment ideas and shows how, using the category of human labor, Marx presents a Materialistic interpretation of dynamic relations between society and environment.

- 


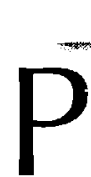

ara muchos ambientalistas y ecologistas contemporáneos, la "naturaleza" o "lo natural" tiene algo de sagrado y por lo tanto debería ser tratado, como todas las cosas sagradas, con reverencia y respeto. Aceptando este punto de vista como correcto, la comprensión de los procesos de la naturaleza debería despertar humildad y por tanto nos obligaría a colocar los planes y logros humanos en un contexto más amplio y a un nivel más elevado. Lo que un filósofo ha identificado como "la arrogancia del humanismo" (Ehrenfield 1981); es decir, darle prioridad a las necesidades del ser humano sobre el resto de la naturaleza se disuelve cuando se estos se enfrentan con la eterna grandeza y belleza de los "planes" y "logros" de la naturaleza misma. Este punto de vista sobre el mundo natural no es raro encontrarlo en importantes círculos de las sociedades del centro de la economía-mundo capitalista. Dicha perspectiva, incluso puede afirmarse, es compartida por la inmensa mayoría de la población de esos países. Sin embargo, ésta responde a un desarrollo relativamente reciente $y$ la referida posición podría haber parecido totalmente fuera de contexto en los siglos XVIII y XIX. Para las gentes y la intelectualidad de esa época, la naturaleza era salvaje, desordenado, incivilizado y cruel. La mayor parte de las elaboraciones intelectuales nacidas del iluminismo europeo compartían esta visión.
En este contexto, para Marx, la comprensión y la explicación de la dinámica capitalista era la más urgente de las prioridades del movimiento obrero y del pensamiento socialista. Obviamente, entonces, la protección y la defensa de la naturaleza no humana no era precisamente parte de su agenda de prioridades intelectuales y políticas. De manera que casi en su totalidad las ideas de Marx, inspiradas en la filosofía de las luces, enfatizan la posibilidad histórica del control racional colectivo de la naturaleza por la sociedad. Para muchos "verdes" este punto de vista moderno es parte del problema y no de su solución ya que propaga la creencia de que no hay limites reales a los logros humanos en términos de su capacidad de ejercer control sobre la naturaleza. Ante esas críticas, la respuesta inicial de varios marxistas y socialistas fue identificar a los movimientos ambientalistas y ecologistas como una respuesta de las clases medias a sus preocupaciones post-materialistas. Sin embargo, en la actualidad varios autores han tratado de aplicar los planteamientos fundamentales del pensamiento de Marx al análisis de la relación existente entre las condiciones ambientales y la lógica capitalista. Es interesante hacer notar que la gran mayoría de ellos se han constituido en los principales portavoces del llamado realismo crítico; el cual es uno de los enfoques más influyentes en los estudios de los problemas ambientales y cuya inspiración proviene particularmente de los 
Manuscritos de 1844 y de la teoría de alienación.

Considerando la importancia que tiene el debate sobre la relación sociedad-naturaleza en el ámbito de las ciencias sociales, el presente trabajo se centra en discutir algunas ideas fundamentales en el pensamiento de Marx para entender las condiciones actuales de dicha relación, al mismo tiempo de presentar su teoría como una consecución y refinamiento de las ideas del iluminismo, y como a partir del uso de categoría del trabajo humano, Marx es capaz de presentar una interpretación materialista de las relaciones dinámicas entre la sociedad y el medio ambiente.

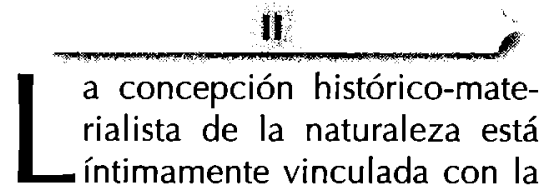
tradición filosófica materialista del iluminismo europeo, el cual constituye en gran medida el fundamento del sistema de Marx. Sin embargo, su materialismo es ajeno al sesgo determinista que caracterizara el mecanicismo cartesiano que cobra especial importancia cuando aborda los problemas de la naturaleza. Este tipo de materialismo estático y ahistórico contrasta con el énfasis que Marx pone en la cualidad dinámica y activa del suyo, en su carácter esencialmente práctico. Tal como señala la primera de las Tesis sobre Feuerbach, la realidad no es, como en el materialismo anterior, un objeto o materia de contemplación, sino "práctica y actividad sensorial humana" (Marx y Engels 1975: 426). El materialismo de Marx adopta de esta manera desde el principio un enfoque relacional que no aprecia tanto la distancia entre el sujeto y el objeto como la interdependencia y conexión de ambos, y de igual modo reconoce en el ser humano a un ser orgánicamente embebido en la realidad práctica que constituye su entorno. Los materialismos precedentes son insuficientes, en la medida en que conciben pasivamente a la materia, $y$ al ser humano en relación a ella, negándose a extraer todas las consecuencias que sus premisas comportan, esto es: en la medida en que son meramente abstractos.

Desde el punto de vista epistemológico, Marx adopta así una posición realista que afirma la existencia independiente de la realidad externa, sin por ello incurrir en ninguna forma de esencialismo, ya que sería contradictorio con su énfasis en la dimensión transformadora de la historia y en la interconexión que existe entre historia social $y$ los procesos de la naturaleza. Para Foster, el de Marx es por ello "un materialismo práctico enraizado en el concepto de praxis (Foster 2000: 15). Y de ahí que, junto con la afirmación de la primacía causal del modo de producción y reproducción del ser humano en el desarrollo de su historia, la vertiente práctica del materialismo filosófico marxista define, según Bhaskar, la afirmación de "el papel constitutivo 
de la mediación transformadora humana en la reproducción y transformación de las formas sociales" (Bhaskar 1984: 520).

Pero el materialismo de Marx tiene también importante implicaciones ontológicas. En este sentido, de nuevo, en palabras de Bhaskar, Marx "afirma la dependencia unilateral del ser social con respecto al ser biológico ( $y$, de forma mas general físico). Baste recordar la afirmación contenida en La ideología alemana: "No es la conciencia la que determina la vida, sino la vida práctica la que determina la conciencia" (Marx y Engels 1970: 26). Tal como lo indica Foster, en esta emergencia biológica de la conciencia individual y de la vida social puede detectarse la honda huella que el evolucionismo darwinista deja en Marx, quien de hecho consideró que la obra de Darwin le proporcionaba la base que su concepción demandaba en la historia natural (Foster 2000: 197). La importancia del evolucionismo es particularmente claro en la obra de Engels (1974), que desarrolla un naturalismo dialéctico en el que el trabajo juega un papel decisivo en la evolución humana. Puede así decir, por que ejemplo: "la mano no es sólo el órgano del trabajo, es también producto de él). Darwin es importante para la concepción histórico-materialista de la naturaleza porque constituye la prueba definitiva y mejor articulada de que la naturaleza no es una entidad inmutable dada de una vez por todas en el principio de los tiempos, sino una realidad dinámica en permanente evolución, que incluye al ser humano como parte de la misma, como producto y factor de ese mismo proceso, proporcionándole con ello el punto de partida para una concepción dialéctica de las relaciones sociedad-naturaleza. Sólo con esta premisa puede explicarse la emergencia humana del seno de la naturaleza.

Porque para Marx el ser humano es parte de la naturaleza, pero a la vez se aparta de ella. En los Manuscritos de 1844 se puede encontrar un desarrollo más detenido de la cuestión. Es precisamente a estos textos a los que con mayor frecuencia recurren aquellos autores interesados en destacar los aspectos de la obra de Marx más compatibles con los fundamentos del ecologismo contemporáneo (Dickens 1992: 64).

Sin embargo, integrando en toda su dimensión los textos de Marx, se puede afirmar que su materialismo adopta una forma naturalista, en la medida que reconoce plenamente la condición natural del ser humano. No obstante, es un naturalismo que no disuelve completamente la diferenciación ser humano-naturaleza, ya que el ser humano depende de la naturaleza como medio de subsistencia y esa misma naturaleza es el objeto insoslayable de su actividad vital, de su trabajo. El ser humano es parte de la naturaleza, pero no es uno con ella: se nutre de ella y sobre ella 
actúa y obra, pero no se confunde con ella. La naturaleza, dice Marx, es el cuerpo inorgánico del ser humano: la necesidad de que ella tiene para sobrevivir la convierte en parte de su cuerpo; sus procesos $y$ estructuras se confunden, estos sí, con los de su organismo. Pero es naturaleza no orgánica: "en cuanto ella misma no cuerpo humano"; no es el ser humano mismo. Este es parte de la naturaleza, es naturaleza. La naturalidad humana acaba implicando la humanización de la naturaleza, una vez que el ser humano comienza transformar como parte de su actividad genérica, pero ello no implica una naturalización del ser humano que anula la diferencia, nacida de la práctica, esto es, del proceso evolutivo, no como producto de una atribución preestablecida que define un destino favorable a la especie humana. Es su vida genérica la que lo distingue del resto de animales, que carecen de ella: la actividad vital del ser humano difiere de la actividad vita! del animal (Marx 1980: 11).

El trabajo distingue al ser humano del resto de especies; que en el trabajo, la actividad productiva es medio de vida, y no un fin. El carácter genérico del ser humano es la actividad libre y consciente; el ser humano, al contrario que los animales, no se separa de su actividad, toma conciencia de la misma (Marx 1980: 111-112).

La clave de la diferenciación reside, por tanto, en la conciencia que el ser humano posee de su pro- pia actividad vital. Por eso, se puede leer en La ideología alemana que "el hombre sólo se distingue del carnero por cuanto su conciencia sustituye al instinto o es el suyo un instinto consciente" (Marx y Engels 1970: 32). El ser humano es algo más que su propio impulso autoconsevador. Es un refinamiento evolutivo de ese impulso: su conciencia. Es esta conciencia la que, a juicio de Marx, lo distingue del animal y lo convierte en ser genérico, Consciente de su propia vida, ésta se convierte en su objeto. Conciencia y libertad producen así vida genérica, si bien resulta en principio algo oscuro ese componente de libertad referido a la actividad vital que permite al ser humano su supervivencia, aunque puede entenderse en referencia al hecho de que el ser humano produce mas de lo que estrictamente necesita, algo que también lo diferencia del resto de los animales.

Más aún: la existencia de una vida genérica, el hecho de que la actividad vital humana se desarrolle bajo el signo de la conciencia y la libertad, supone que las necesidades humanas, a diferencia de las animales son "transformadas en el proceso de su satisfacción de forma distintivamente humana" (Foster 2000: 77). La pertenencia humana a la naturaleza se resuelve en una dependencia material que no excluye la autoreproducción de necesidades y la transformación de las condiciones materiales. Es el signo de la vida genérica. Los seres humanos no tienen un lugar natural 
en el ecosistema, sino que son capaces de adaptarse a muchos entornos: a diferencia de los animales, no cambian su carácter de especie en ese proceso de adaptación, sino que por el contrario lo ejercitan (Grundmann 1991: 100). La singularidad humana trae consigo la existencia y rasgos de su vida genérica. Por ultimo Marx, señala como, en el curso de su desarrollo, la transformación humana de la naturaleza mediante el trabajo da lugar a un nuevo mundo, a una segunda creación específicamente humana, así: "la producción práctica de un mundo objetivo, la elaboración de la naturaleza inorgánica, es la afirmación del hombre como un ser genérico consciente... Por eso precisamente es sólo en la elaboración del mundo objetivo en donde el hombre se afirma realmente como ser genérico." (Marx 1980: 12).

Esta objetivación de la vida genérica del ser humano se alcanza mediante la producción por parte de este ser, de sus propios medios de vida. Con ello, el ser humano produce su propia relación histórica con la naturaleza (Foster 2000: 73). La realidad de la naturaleza es así objeto de apropiación y transformación por parte del ser humano: es humanizada. En última instancia, la vida genérica a la que Marx una $y$ otra vez se refiere expresa que el ser humano es tanto un ser natural como decisivamente un ser social. La transformación de la naturaleza en medio ambiente es la culminación lógica del metabolismo socie- dad-naturaleza en que en El capital se resuelve la vida genérica del ser humano y la autoreproducción de los medios de vida mediante la intervención en la naturaleza de los Manuscritos.

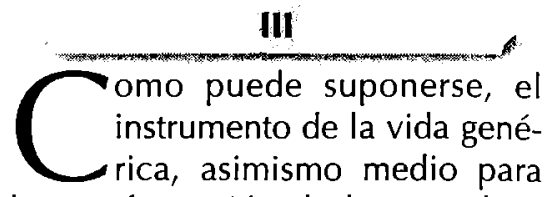

la transformación de la naturaleza que permite su humanización, no es otro que el trabajo. El trabajo humano ocupa un lugar en la concepción histórico-materialista de las relaciones del ser humano y la sociedad con la naturaleza. Mediante el trabajo, la naturaleza es incorporada a la vida humana y social, transformando esa vida en el curso del proceso en el que ella misma es transformada. Actividad social además de individual, el trabajo permite establecer una relación metabólica con la naturaleza. Mediante el trabajo, los seres humanos de apropian de la naturaleza, convirtiéndola en su propio medio ambiente. En este sentido, es una actividad dinámica y constante. El trabajo es la respiración de la sociedad, aquello que la mantiene viva. Esta categoría central en la obra de Marx expresa tanto la base materialista y dialéctica de su concepción de la naturaleza, como su dimensión práctica, en perjuicio, sin embargo de la dimensión cultural y simbólica del proceso de apropiación, dimensiones reducidas a la condición de epifenómenos del proceso material del trabajo. Manifiesta, igualmente, la interdepen- 
dencia y profunda interrelación que, inevitablemente, se establece entre el ser humano y un medio físico en permanente transformación, cuya historia es de hecho incorporada en la historia social lejos por ello de toda concepción ahistórica del mundo natural.

Condición eterna de la vida humana, el trabajo, es, de hecho, su condición de posibilidad. Constituye el fundamento de la vida social, por cuanto es aquella actividad que permite su reproducción mediante la producción de sus medios materiales de vida y la satisfacción de sus necesidades, como tales permanentes, al margen de la historicidad de sus manifestaciones y su posible diversidad. En El capital Marx expone la absoluta necesidad que del trabajo tienen el ser humano y la sociedad en que se organiza, así: "el proceso de trabajo [es] la condición natural eterna de la vida humana, y por tanto, independiente de las formas y modalidades de esta vida y común a todas las formas sociales por igual" (Marx 1978: 136).

Posee el ser humano, por tanto, una necesidad primaria de apropiación de la naturaleza para asegurar su subsistencia: el trabajo es el proceso de tal apropiación transformadora. Aparece el trabajo, así, como fenómeno transhistórico, por más que sus manifestaciones correctas tengan ciertamente, lugar en la historia. De hecho, es el trabajo que hace posible la historia humana. Como escribe Engels el trabajo "es la condición básica y fundamental de toda la vida humana. $Y$ lo es en tal grado que, hasta cierto punto, debemos decir que el trabajo ha creado al propio hombre" (Engels 1974: 59). Sugiere aquí Engels que es por medio del trabajo como el ser humano se ha enfrentado a la naturaleza y la ha dominado, que mediante ese trabajo ha interactuado con ella y la ha transformado, y que es por tanto como consecuencia de ese trabajo que ha evolucionado naturalmente $y$ ha desarrollado cualidades y los rasgos que le permiten diferenciarse del resto de esa naturaleza a la que, después de todo, pertenece. Por ello el trabajo es también un mecanismo adaptativo del ser humano al medio, compartido con resto de organismos en tanto que adaptación, pero diferenciado en su alcance $y$ consecuencias.

Así, el entorno físico del ser humano constituye el objeto del proceso de trabajo. La condición natural del ser humano se ve así refrendada por el hecho de que es en la misma naturaleza donde se encuentra sus medios de subsistencia. Más aún, el trabajo, como escribe Marx en la Critica al Programa de Cotha, "no es más que la manifestación de una fuerza natural, de la fuerza de trabajo del hombre" (Marx y Engels 1975: 10). También el trabajo es un proceso natural, que tiene lugar dentro de la naturaleza, si bien las especificidades de la vida genérica del ser humano, distintas a las del resto de la naturaleza, lo convierten al tiempo en algo distintivamente 
humano. Porque la naturaleza es también el exterior del ser humano, aquello a lo que aplica su trabajo, trabajo que, sin ella, carecería por completo de objeto originario; tal como se expresa en los Manuscritos: "El trabajador no puede crear nada sin la naturaleza, sin el mundo exterior sensible. Esta es la materia en que su trabajo se realiza, en la que obra, en la que y con la que produce" (Marx 1980: 107). En la terminología de El capital, el medio ambiente físico constituye "el objeto general sobre el que versa el trabajo humano" (Marx 1978: 131).

Esta dependencia del trabajo respecto de su objeto no se manifiesta únicamente en el carácter preestablecido del mismo, sino también en la forma de las concretas condiciones naturales sobre las que ese trabajo, considerado ya concreta y no abstractamente va aplicarse. Las características del entorno físico sobre las que el proceso de trabajo tiene lugar condicionan sus posibles resultados de éste. Marx es consciente, no obstante, de que el carácter dialéctico de la relación sociedad-naturaleza exige tener en cuenta los medios materiales o de trabajo de que esa sociedad se sirve para apropiarse de su medio natural, razón por la cual los rasgos de éste, efectivamente condicionan, pero no determinan el curso del proceso de trabajo. De ahí que el valor no proceda del trabajo aisladamente considerado, ni únicamente de la naturaleza que constituye su objeto, sino de su combinación. Mas concretamente, en la transformación de la naturaleza mediante el trabajo la que se constituye en fuente de valor y riqueza; más que la naturaleza en su estado original, por tanto, la naturaleza transformada, adaptada a las necesidades humanas. Solo la aplicación del trabajo a la naturaleza convierte a ésta en valiosa, igual que sólo su previa existencia hace posible su transformación (Marx 1978: 10).

La transformación en que el trabajo consiste humaniza la naturaleza, supone su apropiación por el ser humano. Una vez más, el trabajo se revela como condición de posibilidad de la producción por el ser humano de sus medios de vida, ya que la naturaleza en su estado original no sirve a sus propósitos. Solo a través de la transformación adquiere la naturaleza, que existe con independencia del ser humano, sus cualidades y su significado social (Young 1984: 563). Como acertadamente lo plantea Schmidt (1971: 195), "el trabajo es, en un solo acto, la destrucción de las cosas como inmediatas y su restauración como mediatas".

Pero el proceso de trabajo, no sólo da lugar a la transformación de su objeto; también su sujeto es transformado a lo largo de su desarrollo. Al transformar la naturaleza mediante el trabajo, el ser humano se transforma igualmente. La transformación del entorno produce su efecto en quien la lleva a cabo, con lo que aquélla no es unidireccional, sino recíproca. Por estar ambos, sociedad y naturaleza, sometidos a un proceso de transformación, y al existir la posibilidad misma del cambio, el proceso de tra- 
bajo no podía dejar de producir ese efecto. La transformación de la naturaleza por el ser humano es asimismo la transformación del ser humano por la naturaleza. Y el proceso de trabajo, que constituye el vinculo sociedad y naturaleza, es el desencadenante de esa reciproca transformación; es el origen de la absorción de la historia natural por la historia social. Este carácter recíprocamente transformador del proceso de trabajo proporciona a la postre un argumento más en favor de la supresión de toda barrera entre sociedad y naturaleza, entendidos como ámbitos separados y mutuamente aislados.

Tanto la relación del ser humano con la naturaleza, como el proceso de trabajo, poseen un carácter eminentemente social. Por una parte, en la medida que está ausente en Marx toda la concepción burguesa del vinculo del individuo con la sociedad, el proceso de producción es social en la medida en que las relaciones ser humano-naturaleza corresponden a relaciones sociales definidas y una división del trabajo ya dada, mientras que por otra, el poder del trabajo es una fuerza social en el sentido de que contribuye a la reproducción social y su reproducción y cambio son en sí mismos procesos sociales (Burkett 1999:50; Marx y Engels 1970: 30).

\section{IV}

n Marx, la indudable presencia de la humanización apropiadora del entorno remite directamente a la postura acerca de la "dominación" de la naturaleza. Las relaciones del ser humano con la naturaleza, que son en sí mismas relaciones sociales, se desarrollan dialécticamente, de la contradicción entre ser humano naturaleza cuyas obstáculos aquél tiene que superar para realizarse como especie. La adaptación del ser humano a su entorno físico requiere su "dominación" (conocimiento, transformación). Esta relación es, como en el proceso de trabajo, una condición igualmente eterna de la existencia humana en el mundo. Y aunque la condición natural del ser humano impediría hablar de, en principio, de dominio de la naturaleza, no es menos cierto que el ser humano, sin dejar de pertenecer a ella, se diferencia de la misma en virtud de las especificidades de su vida genérica, dando lugar a una separación, si no orgánica ni biológica, si epistemológica y práctica, entre el ser humano y la sociedad, y el mundo natural de otro. Marx establece una relación directa entre dominio de la naturaleza externa y emancipación del ser humano, en la que aquél es concebido como medio para la ' consecución de ésta.

Por tanto, el ser humano es el soberano de la naturaleza a la que el mismo pertenece $y$ de cuyo inicial dominio debe deshacerse. En este sentido, el tipo de relación que en cada forma social concreta establezca con la naturaleza está en función, a su vez, del grado de control que de la misma alcance; por eso se puede leer en La ideo- 
logía alemana que la naturaleza se enfrenta inicialmente al ser humano como poder extraño y omnipotente, ante el que los seres humanos se comportan de un modo puramente animal, desarrollando por tanto, "una conciencia puramente animal de la naturaleza", esto es, una "religión natural" (Marx y Engels 1970:31). Sólo el progresivo dominio de la naturaleza desplazará esa religión, sustituyéndola por el conocimiento y el poder sobre ella. Mientras que el sometimiento de la naturaleza hace permanecer al ser humano en un estado de necesidad, sólo su dominio creará las condiciones necesarias para el acceso a uno de libertad. El ser humano no debe estar sometido "a las circunstancias exteriores", sino que por el contrario pasa a ser "soberano de dichas circunstancias". La dominación de la naturaleza resulta ser una condición necesaria para la emancipación humana, lo que lleva a Marx a adoptar una actitud antropocéntrica y de control hacia las condiciones medioambientales (Harvey 1996: 126). Sobre todo, la naturaleza aparece en Marx como el medio de realización del desarrollo del ser humano (Giddens 1981: 59). La idea marxista de la naturaleza y de las relaciones sociales con ella sigue fielmente en este punto, la tradición del iluminismo europeo.

Para dominar la naturaleza externa, el ser humano debe apropiársela y subordinarla a sus fines. El trabajo y la tecnología, como herramienta de éste, constituye la relación del ser humano con la naturaleza: mediante el trabajo, el ser humano se apropia de la naturaleza y la transforma. La humanización que esta transformación tiene como uno de sus fines es el dominio de la naturaleza. Así, como señala Grundmann, Marx define la relación ser humano-naturaleza como una relación dialéctica, donde ser humano/naturaleza son sujeto/objeto: la dominación humana de la naturaleza es su apropiación práctica de acuerdo a las necesidades humanas (Grundmann 1988: 6-7). Esta dominación, en todo caso, no puede lograrse prescindiendo de las leyes de la naturaleza a la que se somete. Siguiendo a Bacon, Marx es consciente de que es la utilización para provecho humano de esas leyes, que el conocimiento científico revela y sus aplicaciones prácticas permiten controlar, lo que hace posible ese dominio, no su desconocimiento.

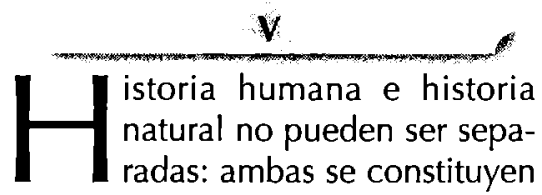
en historia social del ser humano y la naturaleza. Debe recordarse que es precisamente la penetración humana en la naturaleza, realizada con fines autorreproductivos $y$ emancipatorios, la que hace nacer la historia. La concepción materialista de la naturaleza se funde aquí con la concepción materialista de la historia, de modo que la intrínseca relación humana con la naturaleza es el fundamento constante del 
desarrollo histórico, como Marx lo indica: "El primer hecho histórico es, por consiguiente, la producción de los medios indispensables para la satisfacción de estas necesidades, es decir, la producción de la vida material misma..." (Marx y Engels 1970: 28). Al respecto, Foster subraya acertadamente el sentido de esta concepción: lo que Marx señala es que los seres humanos "producen su propia relación histórica con la naturaleza en gran medida mediante la producción de sus medios de subsistencia" (Foster 2000: 73). La práctica histórica de los seres humanos se constituye en conexión, en constante incremento, entre dos áreas aparentemente separadas de la realidad: sociedad y naturaleza (Schmidt 1971: 50). Así, la historia social comprende la historia natural, porque la independencia originaria de ésta se ve progresivamente disminuida, a medida que el ser humano se apropia del mundo natural y lo transforma; aún cuando ontológicamente la naturaleza no siga existiendo autónomamente, la evolución de sus formas se ve afectada por la acción adaptativa de los seres humanos, cuya evolución, a su vez, así como la de sus formas sociales, se ve afectada en este proceso. La historia se concibe así como la progresiva humanización de la naturaleza junto a la que tiene también lugar la naturalización de la humanidad, en el sentido de realización del ser humano como especie, mediante el desarrollo de la vida genérica en la forma de una actividad productiva libre y universal.
En este aspecto, el materialismo histórico puede entenderse como una visión "naturalista" de la vida social, en la que la historia humana aparece como el producto de la evolución natural de lo que, a fin de cuentas, no es más que una parte de la naturaleza: el ser humano. $Y$ así es, naturalmente. Pero no hay que pasar por alto que, en el curso de esa progresiva humanización de la naturaleza, la producción social de los medios de subsistencia y la organización de la satisfacción de las necesidades, la sociedad cobra autonomía respecto de la naturale$z a$, en el sentido de que los requerimientos naturales y los límites de la producción no vienen ya naturalmente dados, sino que son conformados por las relaciones sociales de producción (Burkett 1999:30). Es el sello de la vida genérica. De ahí que el sustrato materialista, de intercambio físico y recíproca influencia, entre la sociedad y la naturaleza, no autorice a considerar la primera un subproducto o epifenómeno de la segunda; si lo que distingue al ser humano como ser genérico es la universalidad, conciencia y libertad cón que produce sus medios de subsistencia y organiza la satisfacción de sus necesidades naturales, es su capacidad para convertir la historia natural en parte de la historia social lo que ha de señalarse, $y$ no contrariamente, la reducción de la historia social a episodio de la historia natural.

El materialismo histórico, que en sí mismo supone la adopción de 
un enfoque realista de la naturaleza, no puede sin embargo, identificarse con las formas ingenuas de realismo con las que la naturaleza aparece como esencia ahistórica e inmutable. La historicidad de la naturaleza que las investigaciones de Darwin vinieron a confirmar, en la opinión de Marx, eliminan esa posibilidad. La combinación de la misma con la historicidad social da lugar a una relación sociedad-naturaleza dinámica y cambiante, donde ambas están en constante transformación y mutua influencia. El realismo de Marx es pues un realismo ontológico, que afirma la existencia independiente de los procesos y estructuras causales de la naturaleza a un nivel profundo. La interpenetración de sociedad y naturaleza, la fusión de historia social e historia natural, apuntan en la misma dirección: el carácter socio-histórico de la naturaleza. No hay una naturaleza prístina, separada del ser humano y definida por su independencia respecto a la acción transformadora de éste. Marx lo niega explícitamente cuando arremete contra el materialismo abstracto de Feuerbach: "Es cierto que queda en pie, en ello, la prioridad de la naturaleza exterior y que todo esto no es aplicable al hombre originario, creado por generatio aequivoca, pero esta diferencia solo tiene sentido siempre y cuando se considere el hombre como algo distinto de la naturaleza "(Marx y Engels 1970: 48).

La naturaleza inmutable no es más que un mito que trata de ocultar la realidad dinámica de unas relaciones sociedad-naturaleza caracterizadas por el cambio y la progresiva separación de toda frontera entre dos ámbitos, que en realidad, son uno solo. Las mismas apariencias naturales ocultan una realidad bien distinta, consecuencia de la unidad de historia natural e historia humana.

Para Marx (1978: 134), también lo natural es un producto de la historia, del proceso de trabajo y de las relaciones sociales que lo organizan y que, por ello, abarcan las relaciones del ser humano con la naturaleza. El "mundo sensible" no es algo "directamente dado desde toda una eternidad y constantemente igual a si mismo, sino el producto de una industria y del estado social, en el sentido que es un producto histórico (Marx y Engels 1970: 47), como lo es, en consecuencial, la creación de necesidades y la organización social orientada a su satisfacción (Marx y Engels 1970: 28-29, 83). Tanto la sociedad como la naturaleza son, de esta manera, productos de la historia, en el sentido de no permanecer dados de una vez por todas, sino de resultar de un constante proceso de cambio. Al suponer la historia la incorporación de la naturaleza, la relación entre estos dos ámbitos se intensifica, $y$ ese proceso es el reflejo de una interrelación constante.

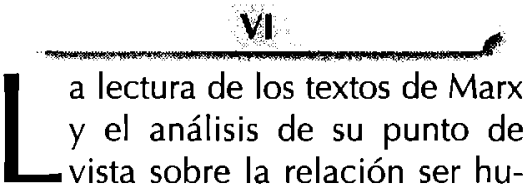


mano-sociedad-naturaleza, no sólo nos permite sentar las bases para una comprensión adecuada de la naturaleza y de las relaciones sociales con el medio físico, sino que constituye también un importante punto de partida para corregir las concepciones bien intencionadas, pero en muchos casos, ingenuas, actualmente en boga sobre el mundo natural y el mundo social. De ahí que sólo a partir de una comprensión materialista y realista será posible brindar una explicación adecuada de la crisis ecológica, de su solución y de la posibilidad de construir un futuro económico, político y culturalmente sustentable para las próximas generaciones.

Así, de acuerdo con la teoría de Marx, la realización de la especie

\section{Referencias}

- Benton, T. (1993) “Ecology, Socialism and the Mastery of Nature: A Reply to Reiner Gundmann", en New Left Review 194. 55-74.

- Bhaskar, R. (1984) "Materialismo", en T. Bottomore (ed.) Diccionario del Pensamiento Marxista. Madrid: Tecnos.

- Burkett, P. (1999) Marx and Nature: A Red and Green Perspectives. Londres: MacMillan.

- Dickens, P. (1992) Society and Nature. Towards a Green Social Theory. London: Harvester. humana mediante su vida genérica sigue cifrándose en la progresiva transformación y apropiación del mundo natural, pero deberá seguir una lógica diferente a la impuesta por las necesidades de la acumulación capitalista; lo que implica una reformulación crítica a la racionalidad de la modernidad burguesa y su visión acerca de la relación entre sociedad y naturaleza. La "plena unidad esencial", como reconoce Benton (1993) no oculta la permanencia del proceso asimilativo de la naturaleza por el ser humano. Resulta así que la naturalización de la humanidad a la que Marx se refiere está vinculada a la completa realización de su vida de especie o vida genérica, que consiste precisamente en la humanización de la naturaleza.

- Ehrenfield, D. (1981) The Arrogance of Humanism. New York: Oxford University Press.

- Engels, F. (1974) Introducción a la dialéctica de la naturaleza. El papel del trabajo en la transformación del mono en hombre. Madrid: Ayuso.

- Foster, J. B. (2000) Marx's Ecology. Nueva York: Monthly Review Press.

- Giddens, A. (1985) A Contemporary Critique of Historical Materialism. Houndmills: MacMillan. 
Grundmann, R. (1991) "The Ecological Challenge to Marxism", en New Left Review 187. 103-120.

Harvey, D. (1996) Justice, Nature and the Geography of Difference. London: Blackwell.

Marx, K y Engels, F. (1970) La ideología alemana. Barcelona: Grijalbo.

(1975) Obras Escogidas I. Madrid: Akai.
Marx, K. (1978) El capital. Crítica de la Economía Política. México: Fondo de Cultura Económica.

(1980) Manuscritos: Economía y filosofía. Madrid: Alianza.

Schmidt, A. (1971) The Concept of Nature in Marx. Londres: NLB.

Young, R. (1984) "Naturaleza", en T. Bottomore (ed.) Diccionario del Pensamiento Marxista. Madrid: Tecnos. 563-564. 